\title{
Spoof Plasmons in Corrugated Metamaterials
}

\author{
Tatjana Gric $^{\mathrm{a}, \mathrm{b}}$, Michael Cada \\ ${ }^{a}$ Dalhousie University, Halifax, Canada \\ ${ }^{\mathrm{b}}$ Vilnius Gediminas Technical University, Vilnius, Lithuania
}

\begin{abstract}
To obtain the eigenmodes of the electromagnetic field at a periodically corrugated metamaterial we consider a theory of spoof plasmons propagating on metamaterial perforated with planar periodic grooves. We present the rigorous modeling and analysis of surface waves at the boundary of the corrugated metamaterial structure. Particularly, we pay special attention to the ways of controlling the properties of surface waves. Moreover, we analyze the dependence of the dispersion characteristics on the metamaterial design.
\end{abstract}

Keywords:Surface plasmons; Surface waves; Terahertz; Metamaterials

\section{INTRODUCTION}

The surface collective charge density oscillations in addition to volume plasmon modes are allowed by the presence of a boundary in a metal[1].Hybrid photon-plasma excitations, known as surfaceplasmonpolaritons (SPPs) emerge once these surface modes couple to light $[2,3]$.

In a previous paper [4], we provided a detailed analysis of the dispersion relation of SPPs on a periodically corrugated semiconductor surface. As an outcome of this study, a low-loss propagation of spoof SPPs has been achieved by an optimum design of the surface structure. It was found that by increasing the lattice constant or by reducing the groove depth, the investigated structure can provide a low guiding attenuation.

Although the analysis of the grating semiconductor structure provides useful information on how the corrugation affects the dispersion relation of SPP modes, it does not provide the full SPP mode solution, including the presence of the anisotropic material, i. e. hyperbolic metamaterial. The purpose of the present paper is to present such study.

In Section 2we formulate the mathematical mechanism of propagation of plasmons in corrugated metamaterials and give the main steps for the derivation of a perturbative treatment that allows us to obtain the complex propagation parameter and the full propagation characteristics of SPPs supported by lossy metamaterialgratings. The method can be considered to be an extension to semiconductor interfaces of the solution presented in [4] for solving the isotropic problem of the interfaces. In Section 3, we use this method to obtain analytical results that correspond to the hyperbolic metamaterial situations considered in [5, 6]. Our simulations show the dispersion characteristics different from those obtained for SPPs propagating along metallic corrugated interfaces.

\section{Propagation of Plasmons in Corrugated Transparent Conducting Oxides}

Let us consider a corrugated surface in which an array of grooves exists. This structure is shown in Fig. 1. The surface is continuous in the $x$-direction and there are two different materials in the $z-y$ plane: the metamaterial occupies the lower region and above there is a dielectric as a surrounding medium. Such an interface can be modelled as a three-layer structure, consisting of a homogeneous anisotropic layer of a thickness $h$ - see figure 1 - describing corrugations, placed between a metamaterial and a dielectric. It is possible to approximate the central layer as an anisotropic effective medium due to the period $d$ being much smaller than the wavelength of the electromagnetic field. It should be pointed out that the investigation of the optical properties of the metamaterial structure (Fig. 1) is carried out via exact mathematical modeling. 


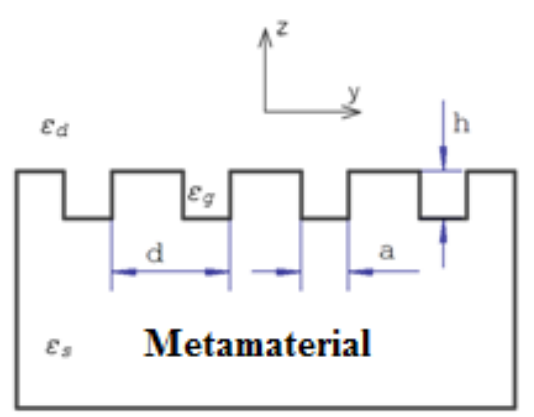

Fig1. Geometry of structured metamaterial surface

In order to find the dielectric parameters of the effective medium, consider a periodic assembly of parallel plates. The effective dielectric constants of such an assembly are as follows [7]

$\varepsilon_{x}=\varepsilon_{z}=\frac{(d-a) \varepsilon_{\perp}+a \varepsilon_{g}}{d}$

$\varepsilon_{y}=\frac{d}{(d-a) / \varepsilon_{\|}+a / \varepsilon_{g}}$

Here, $\varepsilon_{d}$ is the permittivity of the surrounding media, $\varepsilon_{g}$ - is the permittivity of the material, filling the groves, $\varepsilon_{\|}=\left(f_{m_{-} m e t} / \varepsilon_{m_{-} m e t}+f_{d_{-} m e t} / \varepsilon_{d_{-} m e t}\right)$ is the permittivity of the metamaterialwith $f_{m_{-} m e t}=t_{m_{-} m e t} / t$ and $f_{d_{-} m e t}=t_{d_{-} m e t} / t$ being the filling ratio of the metal sheet and dielectric, respectively [8].

By expanding the spoof plasmon wave vector presented in [9], i. e. rearranging it in terms of dielectric parameters of the effective medium, one can obtain the following expression:

$k=\sqrt{\varepsilon_{d} k_{0}^{2}+\left(\frac{\varepsilon_{d}}{\varepsilon_{y}}\right)^{2} k_{g}^{2} \tan ^{2}\left(k_{g} h\right)}$

Here, $k_{0}=2 \pi f / c$ is the vacuum wave vector, $h-$ is the grove depth in the metamaterial, $k_{g}$ is the wave vector of the wave propagating on the grooves defined as [10]:

$k_{g}=k_{0} \sqrt{\varepsilon_{g}}\left(1+\frac{l_{s}(i+1)}{a}\right)^{1 / 2}$

Where $l_{s}=\left(k_{0} \operatorname{Re} \sqrt{-\varepsilon_{\perp}}\right)^{-1}$ is the skin depth with $\varepsilon_{\perp}=f_{m} \varepsilon_{m}+f_{d} \varepsilon_{d}$ [8].

After substituting Eqs. (2) and (4) into Eq. (3) one obtains the following expression of the wave vector

$k=\sqrt{\varepsilon_{d} k_{0}^{2}+\frac{\varepsilon_{g} \varepsilon_{d}^{2} k_{0}^{2} \tan ^{2}\left(\sqrt{\varepsilon_{g}} h k_{0} \sqrt{\gamma}\right)\left(\left(\frac{f_{d_{-} m e t}}{\varepsilon_{d_{-} m e t}}+\frac{f_{m_{-} m e t}}{\varepsilon_{m_{-} m e t}}\right)(a-d)-\frac{a}{\varepsilon_{g}}\right)^{2} \gamma}{d^{2}}}$

Where

$$
\gamma=1+\frac{i+1}{a k_{0} \operatorname{Re}\left(\sqrt{-\varepsilon_{d_{-} m e t} f_{d_{-} m e t}-\varepsilon_{m_{-} \text {met }} f_{m_{-} m e t}}\right)}
$$

Eq. (5) is the dispersion relation for spoof plasmons supported by the corrugated surface of a metamaterial. 


\section{EXAMPLE: HyPERBOLIC METAMATERIAL}

In order to understand the properties of the reported SPPs, we provide a simple example of polaritons in corrugated metamaterials at THz frequencies. To illustrate the properties of SPPs, we plot the wave vector $k$ (Equation (5)) as a function of the frequency. The case of the hyperbolic metamaterial is considered [8]. It is assumed that the structure is surrounded by air, i.e. $\varepsilon_{d}=\varepsilon_{g}=1$.

The dispersion curves of spoof SPPs on structured surfaces with $d=200 \mu \mathrm{m}$ are shown in Figure 3(a). It is of particular interest to analyze the effect of the groove depth on the dispersion curves of spoof SPPs. For this reason, two different groove depths, i.e. $h=3 \mathrm{~d}$ and $2.5 \mathrm{~d}$ are suggested for the study. It should be pointed out that several values of the groove width are considered for each groove depth. As seen from Figure 3, the asymptotic frequency decreases with an increase in groove depth. We thus state that small corrugation amplitudes are needed for obtaining the growth of the asymptotic frequency. This is also the case for the groove width. However, the groove depth drastically affects asymptotic frequency compared to the groove width. We note that by varying the groove depth, it is possible to control the properties of SPPs. The losses of these spoof SPPs as a function of frequency are presented in Figure 3(b). It can be observed that the loss of spoof SPPs grows significantly with an increase in frequency. For instance, in the caseof $a=0.4 d$ and $h=2.5 d$, the attenuation coefficient of the spoof SPPs is $13 \mathrm{~m}^{-1}$ for $f=0.12 \mathrm{THz}$, and it increases up to $38 \mathrm{~m}^{-1}$ for $f=0.13 \mathrm{THz}$ and 80 for $f=$ $0.14 \mathrm{THz}$, respectively. The losses in our study are assigned by investigating a skin effect, i.e. introducing the skin depth, i.e. a measure of how deep electromagnetic waves can penetrate into a material.

The analysis of the effect of the lattice constant (d) on the dispersion of spoofSPPs is of particular importance. In Figure 4(a), we have outlined a situation with the dispersion curves for spoof SPPs on corrugated surfaces with different lattice constants $d=100,150$, and $200 \mu \mathrm{m}$, respectively. The groove parameters are $a=80 \mu \mathrm{m}$ and $h=600 \mu \mathrm{m}$ for all cases. The losses of spoof SPPs for three cases are plotted in Figure 4(b).As seen from Figure 4(b), a smaller lattice constant corresponds to a larger loss of spoof SPPs for a given frequency. As shown in Figure 4(b), the lattice constant drastically affects losses of the spoof SPPs. For a given frequency, an increase of the lattice constantmay results in a significant reduction of the loss of spoof SPPs. With the need for acompact, reliable, and flexible THz system for various applications, a low-loss THzwave guiding system is essential.
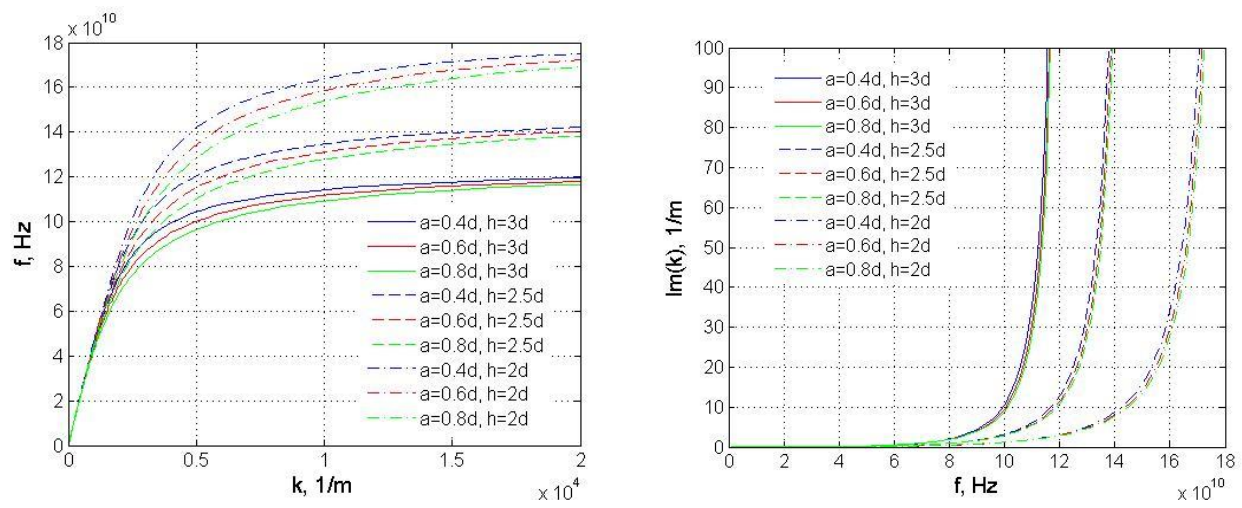

Figure3. (a) Dispersion curves for spoof SPPs. (b) Attenuation coefficients of spoof SPPs, lattice constant $d=200 \mu \mathrm{m}$.
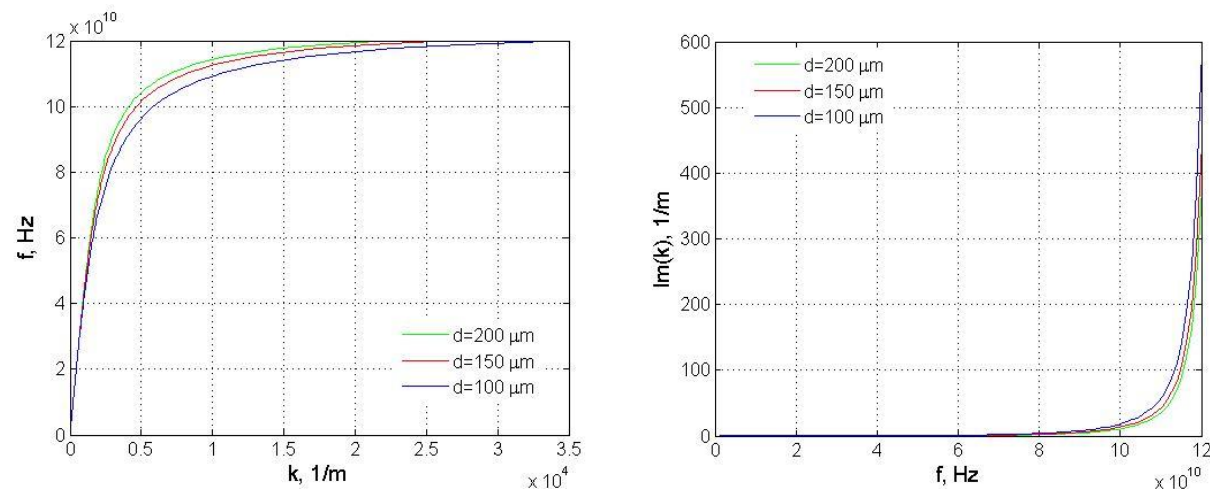
Figure4. Dispersion curves $(a)$ and attenuation coefficients $(b)$ of spoof SPPs for different latticeconstantsd $=$ 100, 150, and $200 \mu \mathrm{m}$, respectively. Parameters of grooves: $a=80 \mu \mathrm{m}$ and $h=600 \mu \mathrm{m}$.

It is of particular interest to analyze the effect of the employed metamaterial on the dispersion curves of spoof SPPs. For this reason four three different metamaterials, i. e. $\mathrm{Au} / \mathrm{HfO}_{2}[11], \mathrm{Au} / \mathrm{Al}_{2} \mathrm{O}_{3}[12]$, $\mathrm{Ag} / \mathrm{MgF}_{2}[13]$ are suggested for the study. As seen from Fig. 5, the smallest asymptotic frequency is achieved employing the metamaterial having dielectric with the smallest permittivity, i. e. $\mathrm{HfO}_{2}$. We can imagine that by changing the dielectric, i. e. by considering a metamaterial with appropriate plasma frequency, the resonant frequencies can be controlled within a broad range of values, thus tailoring the structure for a desired spectral region.
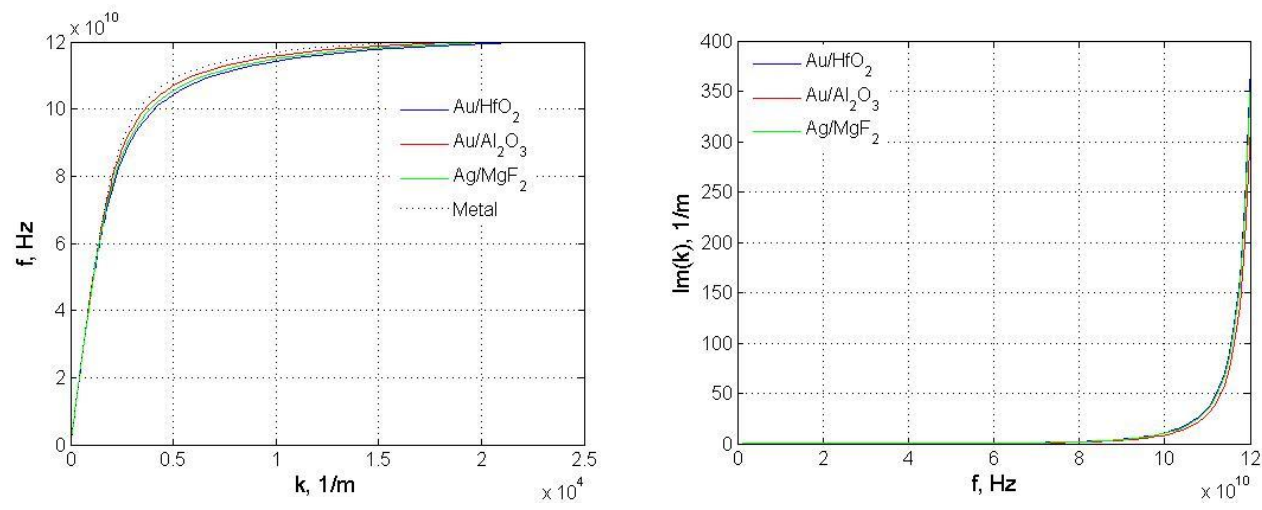

Figure5.Dispersion curves (a) and attenuation coefficients (b) of spoof SPPs for different metamaterials. Lattice constant $d=200 \mu \mathrm{m}$.

\section{Conclusion}

In conclusion, we have introduced a perturbative treatment to find propagation constants associated to SPPs modes propagating along the corrugated metamaterial interface. The methodcan be regarded as an extension to semi conductor inters faces presented in [4]. Moreover, the proposed methodology has been used to investigate how a rectangular corrugation affects the propagation constants. The simulation results presented here corroborate the conclusions obtainedthrough an analysis based on the study of isotropic semiconductor grating interface [4] and allow us to provide the vivid demonstrations of phenomena occurring in metamaterial interfaces, such as the decrease of the asymptotic frequency.

\section{REFERENCES}

[1] R. H. Ritchie, Plasma losses by fast electrons in thin films, Phys. Rev. 106 (1957) 874.

[2] V. M. Agranovich, D. L. Mills, Surface Polaritons: Electromagnetic Waves at Surfaces and Interfaces, $1^{\text {st }}$ Ed., North-Holland, Amsterdam, 1982.

[3] E. N. Economou, Surface plasmons in thin films, Phy. Rev. 182 (1969) 539.

[4] T. Gric, M.S. Wartak, M. Cada, J.J. Wood, O. Hess, J. Pistora,Spoof plasmons in corrugated semiconductors, J. Electromagnet. Wave 29 (2015) 1899-1907.

[5] A.Poddubny,I.Iorsh,P.Belov, Y.Kivshar, Hyperbolic metamaterials, Nat. Photonics 7 (2013) 948-957.

[6] L. Ferrari, C. Wu, D. Lepage, X. Zhang, Z. Liu,Hyperbolicmetamaterials and their applications, Prog. Quant. Electron. 40 (2015) 1-40.

[7] M. Born, E. Wolf, Principles of Optics (Cambridge, 1999).

[8] Y.Xiang, J.Guo, X.Dai, S.Wen, D.Tang,Engineered surface Bloch waves in grapheme-based hyperbolic metamaterials, Opt. Express 22 (2014) 3054-3062.

[9] A.Rusina, M.Durach, M. I.Stockman, Theory of spoof plasmons in real metals, Appl. Phys. A 100 (2010) 375-378.

[10] A.Rusina, M.Durach, K. A.Nelson, M. I.Stockman,Nanoconcentration of terahertz radiation in plasmonic waveguides, Opt. Express 16 (2008) 18576-18589.

[11] I. Iorsh, A. Orlov, P. Belov, Y. Kivshar, Interface modes in nanostructured metal-dielectric metamaterials, Appl. Phys. Lett. 99 (2011) 151914.

[12] J. Kim, V. P. Drachev, Z. Jacob, G. V. Naik, A. Boltasseva, E. E. Narimanov, V. M. Shalaev, Improving the radiative decay rate for dye molecules with hyperbolic metamaterials, Opt. Express 20 (2012) 8100-8116. 
[13] T. U. Tumkur, Lei Gu, J. K. Kitur, E. E. Narimanov, M. A. Noginov, Control of absorption with hyperbolic metamaterials, Appl. Phys. Lett. 100 (2012) 161103.

\section{AUTHORS' BIOGRAPHY}



Prof. Tatjana Gric, Dr.Gric's research career has been focused on the investigation of waveguide devices (waveguide modulators, filters etc.), namely on proposing their electrodynamical analysis. Another major goal of her studies is plasmonics as the examination of the interaction between electromagnetic field and free electrons in a metal. Moreover, the broad scope of research carried out by Dr.Gric has included investigations into the new fascinating properties of novel materials. Dr.Gric also has a record of effective teaching in the rank of Associate Professor. Dr.Gric has published extensively in her field of investigation with more than 25 peer-reviewed papers in top journals in physics, electrodynamics, and optics. It is worth noting that her recent publication rate is getting even higher with her being the first author.

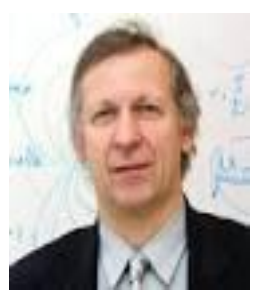

Prof. Michael Cada, Dr.Cada studied with A.M. Prokchorov, a Nobel Prize Laureate for Physics. A former Canada Research Chair in Integrated Active Photonics, he has attracted more than $\mathrm{C} \$ 10$ million in research funds; published more than 250 refereed articles, conference papers and industrial reports; supervised more than 200 highly qualified personnel - and set up a high-tech company. Dr.Cada is a principal investigator in a European Union nanotechnology training project that has recently attracted more than $\mathrm{C} \$ 2$ million in funding. 